\title{
MODELO DE RESPONSABILIDAD SOCIAL PARA LA EMPRESA ELITE DEL SECTOR AUTOMOTRIZ EN LA CIUDAD DE CUENCA, ECUADOR
}

\author{
Jéssica Paola Moyano Reino* \\ https://orcid.org/0000-0003-1319-1080 \\ Celio Froilán Andrade Cordero** \\ https://orcid.org/0000-0003-2660-6783 \\ Mariuxi Jasmin Carreño Godoy*** \\ https://orcid.org/0000-0002-4809-2528
}

\section{RECIBIDO: Septiembre 2019 / ACEPTADO: Noviembre 2019 / PUBLICADO: Enero 2020}

Como citar: Moyano Reino, Jessica; Andrade Cordero, Celio; Carreño Godoy, Mariuxi. (2020). Modelo de Responsabilidad Social para la empresa Elite del sector automotriz en la ciudad de Cuenca, Ecuador. Telos: revista de Estudios Interdisciplinarios en Ciencias Sociales, 22 (1), Venezuela. (Pp. 173183).

DOI: www.doi.org/10.36390/telos221.12

\section{RESUMEN}

El mercado automotriz en la ciudad de Cuenca, se encuentra en lugares con alto flujo de clientes que demandan servicios automotrices, que van desde el mantenimiento regular hasta la venta de repuestos, ofertando servicios adecuados y oportunos. El presente estudio hace énfasis en la problemática que existe en estas zonas como son: la falta de comunicación oportuna entre la empresa y sus clientes, provocando inconvenientes en el ambiente laboral, por ende, se determinó analizar desde la perspectiva de la responsabilidad social, planteando como objetivo diseñar un modelo de Responsabilidad Social que mejore la calidad de atención a los consumidores, ofreciendo servicios oportunos y de calidad, apegados a valores éticos, los siguientes autores dan relevancia al estudio, entre ellos, Aguilera (2012); Feldman y Reficco (2015); Guerrero y Sandoval (2011), entre otros. Desde el plano metodológico, el tipo de investigación es orden descriptivo con enfoque cualitativo, apoyada en el método deductivo. Los resultados indican que asumir enfoques de responsabilidad social orientado a los consumidores generará un ambiente armónico con el cliente y los trabajadores, generando ambientes propicios donde la credibilidad y fidelización de los clientes fortaleza el plano productivo y sustentable de la empresa. Se concluye, que, al asumir este nuevo enfoque, la empresa logrará una comunicación fluida, activa y confiable, elevará la imagen corporativa de la empresa, y podrá definir estrategias de comunicación que permitirán mejorar el ambiente laboral.

Palabras Clave: Responsabilidad social, consumidores, sector automotriz

\footnotetext{
* Contador Público Auditor. Correo: paola.reino@hotmail.com

** Magister en administración de empresas. Universidad Católica de Cuenca, Ecuador. Responsable del Centro de Capacitación y Actualización Profesional. E-mail: candrade@ucacue.edu.ec

*** Administrador de Negocios. Correo: mariuxijasmincarrenogodoy@gmail.com
} 


\title{
Social Responsibility Model for the Elite company of the automotive sector in the city of Cuenca, Ecuador
}

\begin{abstract}
The automotive market in the city of Cuenca, is in places with high flow of customers who demand automotive services, ranging from regular maintenance to the sale of spare parts, offering adequate and timely services. The present study emphasizes the problems that exist in these areas as they are: the lack of timely communication between the company and its clients, causing inconveniences in the work environment, therefore, it was determined to analyze from the perspective of social responsibility, aiming to design a model of Social Responsibility that improves the quality of care to consumers, offering timely and quality services, attached to ethical values, the following authors give relevance to the study, including Aguilera (2012); Feldman and Reficco (2015); Guerrero and Sandoval (2011), among others. From the methodological point of view, the type of research is descriptive order with a qualitative approach, supported by the deductive method. The results indicate that assuming consumer-oriented social responsibility approaches will generate a harmonious environment with the client and workers, generating favorable environments where credibility and customer loyalty strengthens the productive and sustainable plan of the company. It is concluded that, by assuming this new approach, the company will achieve a fluid, active and reliable communication, will elevate the corporate image of the company, and will be able to define communication strategies that will improve the working environment.
\end{abstract}

Keywords: Social responsibility, consumers, automotive sector

\section{Introducción}

La Responsabilidad Social desde la óptica de los consumidores, exige respeto entre la empresa y los clientes; la primera con obligaciones esenciales, y los segundos con derechos básicos. Bajo estos argumentos, la competitividad de los mercados busca mejorar patrones de conducta que establezcan la relación con los consumidores, teniendo como prioridad la generación de un ambiente laboral de tolerancia, con la aplicación de valores y principios éticos y la visión de satisfacer ambas necesidades.

En este sentido, Aguilera (2012:2) sostiene que la responsabilidad social empresarial actúa como un agente clave que impulsa el crecimiento de la empresa, ya que favorece toda iniciativa que se origina, facilitando el posicionamiento de su marca, renovando la imagen corporativa, capturando la preferencia y la lealtad de los clientes y promoviendo la perfecta armonía entre la empresa y la comunidad en la que opera.

Este enfoque en la actualidad, es aplicable a todas las organizaciones, estudiándola en esta oportunidad en el sector automotriz., La empresa Automecánica Elite ubicado en la ciudad de Cuenca desde el año 2014, se ha dedicado a la reparación de vehículos y venta de repuestos de los mismos, enfocándose en brindar un servicio adecuado y oportuno, pero se ha detectado inconvenientes, en la relación de empleados y clientes. En este caso, los consumidores manifestaban no recibir información exacta sobre el servicio prestado por la empresa, 
desconociendo detalles asociados al servicio contratado (tiempos de reparación y entrega del vehículo fundamentalmente).

De igual forma, existe desarticulación entre el departamento de repuestos y los clientes durante procesos clave como la facturación, en el que se pudieron verificar decisiones tomadas por la empresa que no eran comunicadas al cliente con anterioridad, afectando económicamente al cliente. Bajo estas circunstancias, se plantea indagar sobre las responsabilidades de la empresa para con los clientes o consumidores que demandan los servicios ofrecidos, esto con la finalidad de precisar aspectos clave de mejora, que pudieran orientar al diseño de modelo de Responsabilidad Social que optimice la calidad de atención a clientes o usuarios de la empresa Auto mecánica Élite.

El cuidado y atención adecuado de los clientes, debe potenciarse en función del cumplimiento e internalización de valores éticos por parte de empleados y clientes, para ofrecer servicios oportunos, eficientes y sustentables, acompañados de productos de calidad. La idea es generar valor económico, ambiental y social significativo para la empresa.

Al respecto, plantea Carrol (1979:85), citado por Garavito (2008), que, en un modelo de comportamiento social, las empresas valoran la dimensión económica como una de las más importantes, sin embargo, existen otras dimensiones a tomar en cuenta, entre las que destaca el comportamiento de la empresa, en función de otras dimensiones como la filosofía empresarial, la respuesta de la empresa a su entorno y la manera como esta toma en cuenta los intereses de sus miembros y de la sociedad.

Desde el plano metodológico, se asume una investigación de alcance descriptiva, que especifica propiedades, características y perfiles de personas, grupos, comunidades, procesos, objetos 0 cualquier otro fenómeno que se someta a un análisis (Hernández, Fernández y Baptista, 2014:92), en este caso del personal y los servicios ofrecidos por la empresa y el comportamiento de los clientes y usuarios de los mismos. Es decir, únicamente pretenden medir o recoger información de manera independiente o conjunta sobre los conceptos o las variables a las que se refieren, en este caso la responsabilidad social y el comportamiento de los consumidores, clientes o usuarios de los servicios ofrecidos.

El enfoque es cualitativo, en primera instancia, se precian inquietudes básicas sobre la investigación para luego ir perfeccionándolas y respondiéndolas cada una en detalle. La acción indagatoria se mueve de manera dinámica en ambos sentidos: entre los hechos y su interpretación, y resulta un proceso más bien "circular" en el que la secuencia no siempre es la misma, pues varía con cada estudio Hernández, Fernández y Baptista (2014:6). Se apoya en el método deductivo, según plantea Abreu (2014:200), esté método maneja fines que generalmente son categorizados como desarrollo de la teoría y análisis de la teoría respectivamente, es decir permite determinar las características de una realidad particular que se estudia por derivación o resultado de los atributos o enunciados contenidos en proposiciones - leyes científicas de carácter general formuladas con anterioridad, como consecuencias particulares 0 individuales de las inferencias o conclusiones generales aceptadas.

La información levantada se realizó mediante entrevistas al personal del departamento administrativo de la empresa, las cuales se encuentran conformadas por 16 colaboradores, sin embargo, por razones de confidencialidad no se detallarán datos.

Para cerrar, se precisa que la presente investigación se traza como objetivo diseñar un modelo en responsabilidad social para los consumidores de la empresa Auto mecánica Elite, lo cual 
Modelo de Responsabilidad Social para la empresa Elite del sector automotriz en la ciudad de Cuenca, Ecuador

permitirá elevar la imagen corporativa, al igual que establecerá estrategias de comunicación que ayudará a mejorar su ambiente laboral.

\section{Responsabilidad Social: Revisión del estado del arte}

Una empresa que practique la responsabilidad social desde sus niveles jerárquicos altos, involucra directamente a todos sus colaboradores, estableciendo parámetros que generen ambientes laborales propicios para su personal; otorga valor a su imagen como empresa, y confianza tanto de sus clientes, proveedores, empleados, gobierno, por nombrar algunos, fomentando sostenibilidad competitiva.

Barroso (2008), explica que son pocas las organizaciones centradas en ofrecer un bienestar a sus stakeholder (partes interesadas) porque la mayoría de ellas utilizan filantropías para mostrar imágenes corporativas afables, con la idea de generar aumento en ventas. Desde la perspectiva de Aguilera (2012), se establecen medidas de responsabilidad social, bajo dos matices; la primera busca la satisfacción del cliente y de la empresa mediante la actitud consiente de sus actividades; mientras que la segunda, mira a la responsabilidad social como un maquillaje que provoca generación económica, mediante el crecimiento en el mercado y cumplimiento de objetivos corporativos.

La responsabilidad social hacia los clientes, permite establecer medidas de satisfacción de productos o servicios otorgados, mediante la calidad tanto de los bienes como del trato, impulsado a través de políticas, controles de cumplimiento garantizando una trazabilidad exitosa. Por su parte, Feldman y Reficco (2015), proponen una indagación sobre la RSE y el comportamiento de los consumidores, en las cuales precisan que las habilidades empresariales son complementos de la responsabilidad social, ya que impactan en la decisión de consumo solidario y su disposición a comprar con la idea de contribuir a la mejorar social.

En este orden, Alvarado y Schlesinger (2008), examinan la relación que existe entre los componentes de responsabilidad y con sus dimensiones, estableciendo que los elementos económicos, ético-legal y discrecional, determinan una existencia correlacional enmarcada en la imagen y marca, comprobando que estos elementos influyen directamente en su ambiente de laboral, mientras que en la reputación organizacional considera no tener mayor relevancia, estableciendo que no afecta a la organización.

Ávila (2013), por su parte, manifiesta que la aplicación de la responsabilidad social de las pequeñas y medianas empresas para lo cual bosqueja un plan, mediante el manejo de programas dirigidos a los empleados y demás participantes de la organización, con el fin de incorporar estrategias que faciliten el compromiso de la empresa con la sociedad. Hernandez, Abreo y Bohórquez (2018), proponen desarrollar un modelo de (RSE), en la que establecen la incorporación de todos los autores que rodean a la empresa, buscando el bienestar laboral, la motivación en la adopción y concientización de este tipo de prácticas para el resto de organizaciones.

Los nuevos modelos de responsabilidad social, se presentan como desafíos empresariales, debido a que los manejos conservadores se han ligado a un solo parámetro dejando de lado la innovación, por ende, la evolución comercial necesita ideas transformadoras que involucren su compromiso con la sociedad, seguridad, y lo ambiental, siendo herramientas que permitirán transformar y orientar su gestión empresarial. En criterio de Núñez y Carrillo (2006), dan a conocer herramientas de medición a través de indicadores para la responsabilidad social la cual 
diseña parámetros con variables financieras cualitativas y no financieras no cualitativas, brindando una imagen precisa del comportamiento y vinculación al desarrollo de actividades sociales de la organización teniendo en cuenta su actuación en beneficios de la imagen corporativa.

Los resultados positivos en la RSE serven de referencia a la hora de tomar decisiones en la empresa y la implementación en los controles de riesgos. Bigne y Currás (2008), analizan si la responsabilidad social de la empresa es un factor que determine su intención de compra, para lo cual establecen parámetros de medición que le permiten esclarecer que estos comportamientos son innatos dentro de cada organización ya que indirectamente se manejan con principios y valores pegados a la ética por ende se ve reflejado en su imagen corporativa, por lo tanto, ya no utilizan esta mediación.

Los autores mencionados en este estudio, aportan con sus ideas al desarrollo de la investigación, sus conocimientos ayudan a comprender el progreso de la responsabilidad social empresarial, cuyo fin es de brindar oportunidades para que los empresarios sientan la necesidad de implementar compromisos sociales y ambientales.

\section{Empresa de autos Élite: diagnóstico del proceso de comercialización y situación empresarial en función de la responsabilidad social con los consumidores}

Al realizar el análisis de la situación organizacional se permite determinar el estado actual de la empresa, conociendo el manejo de su estrategia, la imagen organizacional frente a sus usuarios y la aplicación correcta de políticas. La conducta del consumidor da apertura a un análisis de actitud que desemboca la organización y los elementos que afectan a su crecimiento en el mercado, la investigación se realizó mediante entrevistas al departamento administrativo, la cual cuenta con 16 colaboradores, por motivo de privacidad, no se citaron apellidos de los empleados entrevistados.

\section{- Proceso de comercialización y situación empresarial}

La empresa Élite desarrolla procesos esenciales para lograr su operatividad y prestar sus servicios a los clientes que lo demandan. Como proceso central, resalta el proceso de comercialización, el cual, durante la investigación realizada, fueron evidentes las falencias 0 debilidades detectadas al momento atender al cliente.

En este sentido, se describe brevemente este proceso que inicia con la recepción del cliente y su vehículo. Se realizó el diagnóstico al mismo y se estableció el presupuesto producto de la avería detectada. Posterior a ello, se consultó el precio de los repuestos requeridos y se informa al cliente, si este está de acuerdo, el vehículo se envía al taller para iniciar el trabajo.

Durante el desarrollo del proceso, pueden verse afectado lo inicialmente planificado, por causas no identificadas en el diagnóstico inicial. En este caso, se comunicó a la recepcionista quien debe comunicarse con el cliente para indicar lo acontecido, precios reales de la reparación del vehículo, entre otros aspectos resaltantes, sin embargo, al no contestar el cliente, se procede con la reparación, apoyados en el costo presuntivo inicial, sin embargo, los costos varían, y al momento de cancelar resulta diferentes valores en perjuicio del consumidor siendo causa de ambiente disfuncional en la organización. Otro proceso prejuicioso, es que, al no conseguir los repuestos para la reparación, no se lo comunica al dueño en seguida extendiendo, el tiempo de restauración del vehículo, y por ende generando descontento en el cliente o usuario. 
Modelo de Responsabilidad Social para la empresa Elite del sector automotriz en la ciudad de Cuenca, Ecuador

En la figura 1, se diagrama la estructura del proceso descrito (figura 1):

Figura 1.

Proceso de comercialización empresa Élite

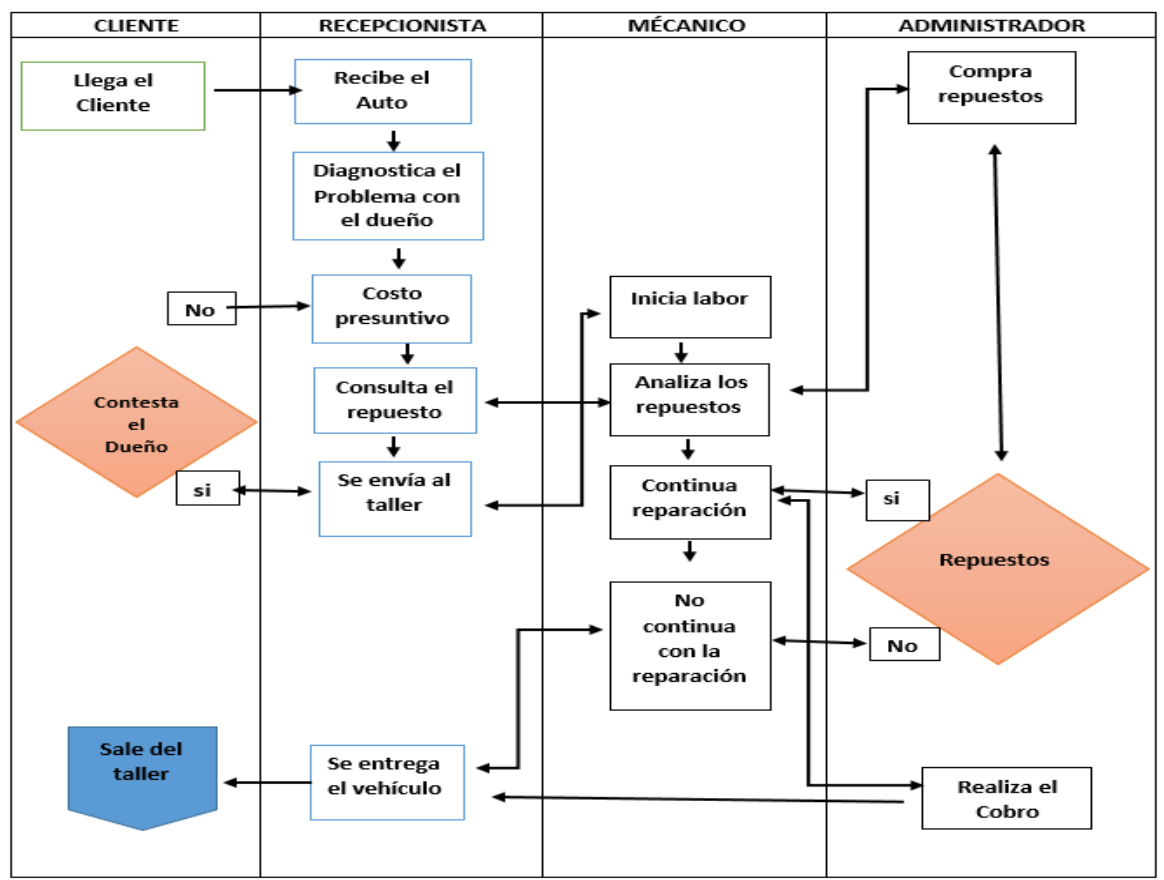

Fuente: Elaboración propia

Según las entrevistas realizadas, se determinó que la responsabilidad social dentro de la Auto mecánica Elite es baja, debido al mal manejo de políticas administrativas, al no prestar importancia a los clientes o usuarios del servicio, mismos que son necesarios para la subsistencia de la organización. Adicionalmente, se considera la importancia que ha restado a la inserción laboral y practicas operacionales justas con el entorno ambiental, mejorar en el ambiente de trabajo, satisfacción del cliente, compromiso con los proveedores, incumpliendo de esta manera con la misión y visión de la empresa en mención.

\section{- Análisis del Comportamiento del consumidor}

Sergueyevna y Mosher (2013), manifiestan que el comportamiento de consumidor hace referencia al análisis de las necesidades y el deseo de los usuarios, relacionado al grado de saacción del individuo.

Las empresas deben contemplar que las políticas que defienden al consumidor, incitan a las organizaciones a cumplir con lineamientos de servicio de la calidad, información transparente, 
Indemnización por daño, entre otros. Ante esta realidad, las entidades tienen la obligación de hacer partícipes a los consumidores dentro de sus programas organizacionales como: participar de promociones, publicidades, y actuar asertivamente frente a las conductas de los usuarios.

En la siguiente tabla se muestra el comportamiento del consumidor según la investigación realizada:

\section{Cuadro 1}

Análisis del Comportamiento del consumidor

\begin{tabular}{|l|l|}
\hline Económica & $\begin{array}{l}\text { Producción de servicios adecuados. } \\
\text { Obtener Beneficios }\end{array}$ \\
\hline Legal & $\begin{array}{l}\text { Cumplir las normativas vigentes } \\
\text { Cumplimiento éticos. }\end{array}$ \\
\hline Socio Ambiental & $\begin{array}{l}\text { Programas que incluyan al consumidor. } \\
\text { Discrecionalidad en las actuaciones de los servicios, sin } \\
\text { afectar el espacio al consumidor. }\end{array}$ \\
\hline
\end{tabular}

Fuente: Elaboración propia

Por su parte, Arellano (2017), analiza la relación existente entre la calidad de productos y los servicios adecuados en la cual determina que la producción debe contar con parámetros satisfactorios para los consumidores, permitiéndole tener ventaja competitiva, por ende, sus clientes sentirán el deseo de optar por estos servicios y liquidarán la cantidad de dinero demando, logrando el aumento económico hacia la empresa y fidelizando clientes.

En el Ecuador hay leyes que hacen referencia a defender los derechos de los consumidores y los cuales las empresas deben poner énfasis en su cumplimiento, por lo cual genera una responsabilidad de acatamiento y se reflejara con la satisfacción de sus clientes, y el art 92 de la Constitución Política del Ecuador (Asamblea Nacional Constituyente, 2008) dispone que:

"La ley establecerá los mecanismos de control de calidad, los procedimientos de defensa del consumidor, la reparación e indemnización por deficiencias, daños y mala calidad de bienes y servicios (...) y las sanciones por la violación de estos derechos" (p.1).

León (2008), manifiesta que la responsabilidad social es un factor que influye al instante de la compra, y la percepción de los consumidores cambian al momento de sentirse tomando en cuenta por parte de las empresas, consideran que las organizaciones deben tomar énfasis en sus opiniones y discreciones siendo factores que influyen en la imagen y reputación, provocando rentabilidad a la organización.

\section{- Indicadores de responsabilidad social con los consumidores}

Los indicadores permiten establecer medidas de control que se ejercen en el cumplimiento de parámetros exigidos por la organización, para lo cual la satisfacción de las exigencias, esclarece la calidad del servicio brindado, no solo enfatizando en el proceso, sino en los resultados brindados por la empresa y satisfaga tanto en servicio como en calidad la prestación brindada. El indicador de Compromiso analiza que los empleados apliquen la ética y la moral en su trabajo, forjando un estímulo de complacencia en la labor realizada, cumpliendo metas y aprovechamiento apropiado de los materiales institucionales. El indicador de resultados positivos califica su agrado hacia la atención de la organización las cuales se ven reflejados en 
Modelo de Responsabilidad Social para la empresa Elite del sector automotriz en la ciudad de Cuenca, Ecuador

su retorno y recomendaciones brindadas, cumpliendo con los objetivos enfocados. Así, Alen y Fraiz (2006), establecen parámetros que permiten determinar la calidad del servicio y la satisfacción del consumidor en la cual se realiza un análisis y explica la relación e influencia entre estos elementos, determinando que la calidad de servicio es un antecedente importante de la satisfacción. García, Carreón y Bustos (2012), analizan indicadores de compromiso laboral, en las cuales miden el grado de cumplimiento en las actividades de los trabajadores de la organización, y su fidelidad hacia la empresa.

\section{Cuadro 2.}

Indicadores de responsabilidad social con los consumidores

\begin{tabular}{|l|l|}
\hline Satisfacción de exigencias & $\begin{array}{l}\text { Se analiza el número de quejas recibidas, eficiencia, } \\
\text { eficacia y la aceptación de la calidad. }\end{array}$ \\
\hline Compromiso del Personal & $\begin{array}{l}\text { Tiempo en el desarrollo del servicio, cumplimiento } \\
\text { de valores y principios organizacionales, análisis de } \\
\text { procesos, usos de recursos. }\end{array}$ \\
\hline Servicio con resultados positivos & $\begin{array}{l}\text { Comentarios positivos, Retorno de usuarios, grado } \\
\text { de cumplimiento de metas, grado de satisfacción de } \\
\text { los usuarios. }\end{array}$ \\
\hline
\end{tabular}

Fuente: Elaboración propia

- Consecuencia de resultados de responsabilidad con los consumidores

Los productos de calidad ayudaran a los usuarios a confiar en la honestidad de la empresa ya que genera credibilidad y mejora la imagen organizacional, sintiéndose satisfecho con lo solicitado, y garantizando una comunicación propicia con los consumidores.

El incremento productivo se deberá a la satisfacción de los usuarios, y al cumplimiento de normas y principios que generan ambientes de confianza, agregando valor a la empresa, cuyo beneficio será la ventaja competitiva construyendo una lista de clientes leales.

Los indicadores organizacionales establecen medidas de conducta de los usuarios en relación al cumplimiento de objetivos bajo un perfil de ética y buena atención al consumidor.

La consecuencia de resultados de responsabilidad permite crear confianza y credibilidad reflejada en el grado de satisfacción del consumidor, da creando un ambiente competitivo ante un mercado de servicio automotriz altamente desarrollado.

\section{- Modelo de responsabilidad social para consumidores}

Luego de la disertación realizada, se diseña un modelo de responsabilidad social para generar grandes oportunidades de negocio y mejorar el servicio a los clientes, dando mayor credibilidad ante la sociedad, favoreciendo claramente su imagen y ampliando la disposición del consumidor, optimizando de esta manera las relaciones con el grupo de interés en mención mejorando el flujo de información, la fiabilidad en nuevas relaciones, e incluso permitiendo reducir costos para brindar mejores productos o servicios y aumentar la productividad.

El modelo de responsabilidad social, permite tener competitividad y mantener una relación ética con los competidores, propiciando credibilidad en el mercado y generando sobre todo una estructura sólida, dando énfasis a una organización consolidada en la atención al consumidor. Guerrero y Sandoval (2011), ejecutaron un modelo de responsabilidad social en la cual identificaron factores que generan balances de buenas prácticas sociales, estableciendo 
indicadores de evaluación y seguimiento de procesos que ayuden a determinar el cumplimiento del modelo.

Finalmente, Koehn y Ulloa (2015), manifiestan que un modelo de responsabilidad social en las empresas ecuatorianas generaría impactos sostenibles a mediano plazo dado que promovería su imagen corporativa a través de estrategias en las cuales se enfaticen el respeto hacia sus clientes, colaboradores y medioambiente.

Cuadro 3.

Modelo de responsabilidad social para consumidores

\begin{tabular}{|l|l|}
\hline Situación Organizacional & $\begin{array}{l}\text { Políticas de la empresa } \\
\text { Análisis de Principios y Valores Corporativos. }\end{array}$ \\
\hline comportamiento los consumidores & $\begin{array}{l}\text { Económica } \\
\text { Legal } \\
\text { Ámbito social }\end{array}$ \\
\hline $\begin{array}{l}\text { Indicadores de responsabilidad social con los } \\
\text { consumidores }\end{array}$ & $\begin{array}{l}\text { Satisfacción de exigencias } \\
\text { Compromiso del Personal } \\
\text { Servicio con resultados satisfactorios }\end{array}$ \\
\hline $\begin{array}{l}\text { Consecuencia de resultados de responsabilidad } \\
\text { con los consumidores }\end{array}$ & $\begin{array}{l}\text { Productos de calidad } \\
\text { Mayor productividad }\end{array}$ \\
\hline
\end{tabular}

Fuente: Elaboración propia

\section{Conclusiones}

Con el estudio propuesto se da cumplimento al objetivo, que fue diseñar un modelo de responsabilidad social para los consumidores de la empresa "Auto mecánica Élite", analizado a través de la medición de sus necesidades, que permitirá, efectuar procesos que generen actividades de manera oportuna, mediante una comunicación organizada y clara enfocándose en la satisfacción de sus clientes.

Este enfoque de responsabilidad social para con sus consumidores, establece parámetros de compromiso de sus trabajadores hacia la empresa, lo cual agregara valor a la organización en el aumento de la productividad. Se debe tener énfasis en que la buena atención provoca mayor credibilidad a la imagen corporativa y confianza en sus consumidores

Al realizar filosofías en las cuales se incluya los aspectos de: calidad de repuestos, transparencia en la información y tiempo de entrega, enfatizando en generar políticas que mejoren la productividad, concurre a la sostenibilidad de la empresa en el tiempo porque establece medidas que conllevan a adoptar hábitos, estrategias y procesos que nos ayuden a minimizar los impactos negativos que se puede generar en la comunidad.

\section{Referencias bibliográficas}

Abreu, José (2014). El Método de la Investigación. Revista: International Journal of Good Conscience. Volumen 9, No 3. (Pp.195-204). Extraído de: http://www.spentamexico.org/v9-n3/A17.9(3)195-204.pdf, consulta: 4/07/19

Aguilera, Adriana (2012). Crecimiento empresarial basado en la Responsabilidad Social. Revista: Pensamiento y Gestión. No32. (Pp.1-32). Colombia. Extraído en: http://www.scielo.org.co/scielo.php?script=sci_abstract\&pid=S1657-

62762012000100002 consulta: $8 / 07 / 19$ 
Modelo de Responsabilidad Social para la empresa Elite del sector automotriz en la ciudad de Cuenca, Ecuador

Alén, María y Fraiz, José (2006). Relación entre la calidad de servicio y la satisfacción del consumidor. Su evaluación en el ámbito del turismo termal. Revista: Investigaciones Europeas de Dirección y Economía de la Empresa. Volumen12, No1. (Pp. 251-272) España, Extraído de: https://www.redalyc.org/pdf/2741/274120878011.pdf, consulta: $17 / 07 / 19$

Alvarado, Alejandro y Schlesinger, María (2008). Dimensionalidad de la responsabilidad social empresarial percibida y sus efectos sobre la imagen y la reputación: una aproximación al modelo de Carroll. Revista: Estudios Gerenciales. Volumen 24. No.108. (Pp.3759) España. Extraído de: https://doi.org/10.1016/S0123-5923(08)70043-3 consulta:4/07/19

Arellano, Hernán (2017) La calidad en el servicio como ventaja competitiva. Revista: Dominio de las ciencias. Volumen3, No2. (Pp:72-83). Extraído de: https://dialnet.unirioja.es/servlet/articulo?codigo=6093282 consulta: 19/07/19

Asamblea Nacional Constituyente (2008). Constitución Política del Ecuador. Asamblea Nacional Constituyente. Ecuador.

Ávila, Flor (2013). Plan de responsabilidad social empresarial: una propuesta para pequeñas y medianas empresas industriales de la provincia de Tundama. Revista: Criterio Libre. Volumen11, No18. (Pp.217-228) Colombia. Extraído de https://doi.org/10.18041/19000642/criteriolibre.2013v11n18.1132 consulta:8/07/19

Barroso, Francisco (2008). La responsabilidad social empresarial Un estudio en cuarenta empresas de la ciudad de Mérida, Yucatán. Revista Sciencie Direct. Volumen31. Colombia. (Pp 373-382). Extraído en http://www.redalyc.org/pdf/395/39522605.pdf, consulta: $18 / 07 / 19$

Bigne, Enrique y Currás, Rafael (2008). ¿Influye la imagen de responsabilidad social en la intención de compra? el papel de la identificación del consumidor con la empresa. Revista; Universia Business Review, No19, (Pp.10-23). España. Extraído de: https://www.redalyc.org/pdf/433/43301901.pdf, consulta: 3/07/19

Extraído de: https://gorila.furb.br/ojs/index.php/universocontabil/article/view/108/67, consulta: 23/07/19

Feldman, Percy y Reficco, Ezequiel (2015). Impacto de la responsabilidad social empresarial en el comportamiento de compra y disposición a pagar de consumidores. Revista Estudios Gerenciales.Volumen31. No137. (373-382) Colombia. Extraído de: https://doi.org/10.1016/j.estger.2015.10.001 consulta: 10/07/19

Garavito, Cecilia (2008). Responsabilidad social empresarial y mercado de trabajo. Revista

Economía.Volumen31. No61.Perú. (Pp.81-104). Extraído en

http://revistas.pucp.edu.pe/index.php/economia/article/view/488/478 consulta: 4/07/19

García, Cruz; Carreón, Javier y Bustos, José (2012) Socialización y compromiso laboral en trabajadores sociales de instituciones públicas y privadas. Revista: Humanismo y Trabajo Social. Volumen11. (Pp. 177-192) México. Extraído de: https://buleria.unileon.es/bitstream/handle/10612/3463/Cruz.pdf?sequence=1\&isAllow ed=y, consulta: 24/07/19

Guerrero, Doris, y Sandoval, Jorge (2011) La responsabilidad social como generadora de valor empresarial: hacia la construcción de un modelo de balance social para las MiPymes. Revista: U.D.C.A Actualidad y Divulgación Científica. Volumen14, No2. 
(Pp.177-186).

Extraído

de:

https://revistas.udca.edu.co/index.php/ruadc/article/view/795, consulta: 15/07/19

Hernández, Angélica; Abreo, Brayan y Bohórquez, Lesley (2018). Programa de Responsabilidad social empresarial: Empresa Global Service y Business S.A.S. Revista: Aibi Revista de Investigación, Administración e Ingeniería, Volumen 6, No. 2. (Pp.2-12). Colombia. Extraído de: https://doi.org/10.15649/2346030X.479, consulta: 26/07/19

Hernández, Roberto; Fernandez, Carlos, y Baptista, María del Pilar (2014). Metodología de la Investigación Científica (sexta edición). Editorial: Mg Geaw Grill. México.

Koehn, Peter y Ulloa, Isidro (2015) El modelo de responsabilidad social empresarial como estrategia en el sector inmobiliario en el Ecuador. Revista: Saber, Ciencia y libertad. Volumen10, No.1. (Pp:101-114) Ecuador. Extraído de: https://doi.org/10.18041/23823240/saber.2015v10n1.875, consulta: 9/07/19

León, Fátima (2008) La percepción de la responsabilidad social empresarial por parte del consumidor. Revista: Visión Gerencial. Volumen7, No1. (Pp. 83-95) Venezuela. Extraído de:

https:

http://erevistas.saber.ula.ve/index.php/visiongerencial/article/view/893

consulta: $5 / 07 / 19$

Núñez, Montserrat y Carrillo, Inmaculada (2006). Propuesta de indicadores de responsabilidad social preventiva. Revista Universo Contabíl, Volumen2, No1. (Pp.90-102). España. Sergueyevna, Natalia y Mosher, Elmer (2013), Teorías motivacionales desde la perspectiva de comportamiento del consumidor. Revista Negotium, Volumen 9, No26. (Pp. 5-18) Venezuela. Extraído de: https://www.redalyc.org/pdf/782/78228464001.pdf, consulta: $5 / 07 / 19$ 